\title{
An Enhanced Texture-Based Feature Extraction Approach for Classification of Biomedical Images of CT-Scan of Lungs
}

\author{
${ }^{1}$ Bharati Vidyapeeth's College of Engineering, Paschim Vihar, New Delhi (India) \\ ${ }^{2}$ Atria Institute of Technology, Bengaluru, Karnataka (India) \\ ${ }^{3}$ Netaji Subhas University of Technology, East Campus, Delhi (India) \\ ${ }^{4}$ Department of Computer Science, University of Oviedo, Oviedo (Spain)
}

Varun Srivastava ${ }^{1}$, Shilpa Gupta ${ }^{1}$, Gopal Chaudhary ${ }^{1}$, Arun Balodi², Manju Khari ${ }^{3}$, Vicente García-Díaz *

Received 14 August 2020 | Accepted 9 October 2020 | Published 2 November 2020

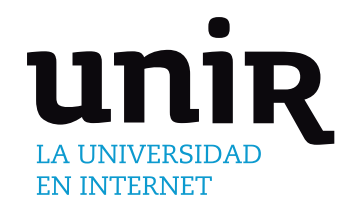

KEYWORDS

ABSTRACT

Content Based Image Retrieval (CBIR) techniques based on texture have gained a lot of popularity in recent times. In the proposed work, a feature vector is obtained by concatenation of features extracted from local mesh peak valley edge pattern (LMePVEP) technique; a dynamic threshold based local mesh ternary pattern technique and texture of the image in five different directions. The concatenated feature vector is then used to classify images of two datasets viz. Emphysema dataset and Early Lung Cancer Action Program (ELCAP) lung database. The proposed framework has improved the accuracy by $12.56 \%, 9.71 \%$ and $7.01 \%$ in average for data set 1 and $9.37 \%, 8.99 \%$ and $7.63 \%$ in average for dataset 2 over three popular algorithms used for image retrieval.

\author{
Image Retrieval, Local \\ Mesh Peak Valley Edge \\ Patterns, Local Patterns, \\ Bio-medical Image \\ Classification, Texture- \\ based Retrieval.
}

DOI: $10.9781 /$ ijimai.2020.11.003

\section{INTRODUCTION}

\begin{abstract}
$\mathrm{C}$ ONTENT based image retrieval (CBIR) relies upon the extraction of features present in an image to fetch related images from a database. Out of the multiple techniques of CBIR based feature extraction, texture-based features are one of the most popular and earliest [1]. Various algorithms like local binary pattern (LBP), local ternary pattern (LTP), local mesh patterns (LMeP), etc. have been proposed to extract texture from a given image for CBIR.

Also, multiple image retrieval techniques based on texture information have been proposed in the literature [2]-[11]. A comparison of state-of-the-art biomedical image retrieval techniques has been given in [5]. The texture-based feature vector has been introduced by the concept of local binary patterns (LBPs). LBP compares the intensity of central pixel to its $P$ neighbours at radius $R$ [12]. If the intensity of neighbour is greater than central pixel then feature vector is assigned a value 1 or otherwise 0 . This constitutes a feature vector of size $P$ which is then multiplied by a weight vector and finally summed up to form the intensity value at that pixel. Similarly, the technique of local ternary pattern is introduced where instead of one threshold (central pixel intensity); two thresholds are used [13]. These techniques are modified by various researchers to propose other texture-based approaches. If the central pixel intensity is compared to neighbours in different directions, we retrieve local mesh ternary patterns [2] or if the edge
\end{abstract}

${ }^{*}$ Corresponding author.

E-mail address: garciavicente@uniovi.es information is incorporated into the texture information, then local maximum edge binary patterns are formed [11].

Recently, researchers have tried to merge texture-based features with other features like frequency-based features, tags, shape based features etc. as well. In this way average retrieval rate (ARR) and average retrieval precision (ARP) are improved to a great deal for medical image retrieval. Authors in [14] presented a retrieval approach in which colour, texture and edge information are used as features. Further Principal Component Analysis (PCA) is applied to reduce these features which are then fed into a support vector machine (SVM) and a fuzzy classifier model (FCM) for statistical similarity measurement. Image retrieval techniques are also very popular for biomedical datasets. Some of them are compared in [15]. Diabetic retinopathy is performed in [16] by calculating LBP for different planes. Edges of an object in the image are detected in red, green and blue planes and the corresponding LBP relationships are extracted for each plane. These LBP feature vectors are then combined to generate the final feature set. In [17], textual and shape-based features are extracted and combined to form the final feature matrix. Authors in [18] generated a visual model of an input biomedical image. A SVM model is generated for classification of that visual model. The visual and semantic similarities are then used to form the feature vector. A combination of text and image texture to form the feature vector for image retrieval has also been proposed in the literature. The associated tags (text) with the images are used along with the texture to extract images in [19], [20] and [21]. Texture-based algorithms have also been extended in many ways. Authors in [22] used RGB components along with luminescence and chrominance components whose texture pattern has been computed. 
A histogram of these texture patterns is then used as a feature vector. In [23], the centre symmetric local binary patterns are calculated and are then combined with co-occurrence matrix calculated in $0,45,90$ and 135 degrees. The feature vector obtained thereby is used for image retrieval. A robust approach is proposed for biomedical image retrieval in [24] using Zernike moments. Spatial and wavelet-based features are combined in [25] to form the feature vector for extraction of similar mammograms. Texture in 0, 45, 90, 135 and 180 degrees are extracted from an image in [26]. Further the technique of local ternary patterns (LTP) is applied in these directions to form the feature vector. A second-order image moments in the local neighbourhood is computed in [27]. A covariance matrix is then computed followed by Eigen value decomposition. The principal Eigen value is then normalized using gray value of the center pixel. A refined histogram is then obtained for image retrieval. A texture block coding-based tree data structure is proposed in [28] for effective image retrieval. The precision, recall and $F$ score were compared and were better as compared to the similar algorithms. Instead of a 2D $3^{*} 3$ block, a $3^{*} 3^{*} 3$ 3D block is used in [29] to extract 3D ternary patterns. These 3D ternary patterns are then used for image retrieval. In [34], authors extracted feature vector by first decomposing an original image using wavelet decomposition for five consecutive times and then used probabilistic principal component analysis to reduce its features. The reduced set of features is classified using feed forward neural network or k nearest neighbour algorithm. A brief overview of various types of cancers and a summary of recent work to identify the extent of possible damage in the corresponding tissue has been discussed in [35].

Recent techniques involve the use of deep convolutional networks for extraction of similar images from biomedical dataset. Few such algorithms are given in [30] and [31] where deep convolutional features are extracted to retrieve similar images. Authors in [32] used wavelet decomposition along with principal component analysis to classify various MR images of brain. In [33], authors proposed a local mesh peak valley edge pattern feature vector to extract similar images from a given image dataset. The algorithm computed $j^{\text {th }}$ order derivatives in forward and backward directions to compute peak and valley texture from an image.

The proposed algorithm is a hybrid of two very popular image retrieval approaches viz. local mesh peak valley edge pattern (LMePVEP) and local mesh ternary pattern (LMeTerP) to retrieve similar images. A new feature set obtained by computing texture in different directions is introduced in this paper and combined with the aforementioned two feature sets. The concatenation of feature set has yielded a very comprehensive feature vector with much more accuracy as compared to LMePVEP and LMeTerP. Thereby the proposed algorithm is found better than two recent biomedical image retrieval algorithms for classification of diseased and healthy patient's images. The paper has been organized in the following manner. Section II discusses the various steps of the proposed methodology. Section III summarizes the results and compares the given algorithm with three other algorithms used for image retrieval. Section IV presents the conclusion for the given work.

\section{MAterials AND Methods}

\section{A. Local Mesh Peak Valley Edge Patterns}

Local mesh peak valley edge pattern (LMePVEP) as given in [33] computes the texture details from a given image in terms of peak and valleys. It compares the central pixel intensity with $P$ neighbors at radius $R$ and calculates the first order gradient in both forward and backward direction. If $\mathrm{I}_{P, R}^{\mathrm{f}}$ represents a first order forward derivative vector, then for central pixel with intensity $\mathrm{I}\left(g_{c}\right)$, the $j^{\text {th }}$ order gradient in forward direction can be computed using eq. (1).

$$
\begin{aligned}
& I_{P, R}^{f_{R}}\left(g_{c}, g_{i}\right)=I(g(\alpha))-I(g(i)) \mathrm{i}=1,2, \ldots \mathrm{P} \\
& \text { where } \alpha=1+\bmod ((\mathrm{i}+\mathrm{P}+\mathrm{j}-1), \mathrm{P})
\end{aligned}
$$

and $\mathrm{j}=1,2 \ldots \mathrm{P} / 2$, which represents the distance for first order derivation

Similarly, $j^{\text {th }}$ order backward derivative $I_{P, R}^{b}$, can be defined as

$$
I^{b}{ }_{P, R}\left(g_{c}, g_{i}\right)=\left\{\begin{array}{l}
I(g(P+i-j))-I g(i) \text { if } j \geq i \\
I(g(i-j))-I g(i) \text { otherwise }
\end{array}\right.
$$

where values of $i$ and $j$ vary as in eq. (1) and eq. (2) respectively.

Local mesh peak valley edge pattern LMePVEP $(\mathrm{P}, \mathrm{R})$ is then calculated using eq. (4) in the form of a column vector of size $1^{*} P$.

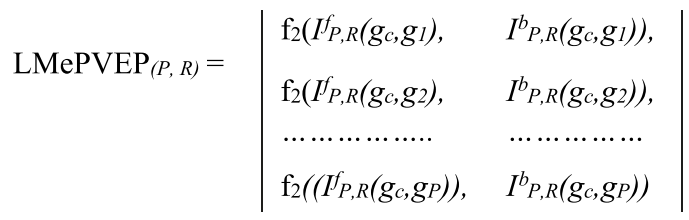

where the function $\mathrm{f}_{2}(\mathrm{x}, \mathrm{y})$ used in eq. (4) is defined as:

$$
\mathrm{f}_{2}(x, y)=\left\{\begin{array}{rr}
1 & \text { if } x>0 \text { and } y>0 \\
2 & \text { if } x<1 \text { and } y<1 \\
0 & \text { otherwise }
\end{array}\right.
$$

Therefore, the possible values of all components of LMePVEP vector can be 0,1 or 2. Fig. 1 represents an illustration for computation of Combined LMePVEP values. The illustration considers a 3x3 block of original image and the LMePVEP values for 8 neighbors at radius $\mathrm{R}$ as 1 are computed thereby.

\begin{tabular}{|l|l|l|}
\hline 7 & 2 & 4 \\
\hline 9 & 6 & 8 \\
\hline 1 & 4 & 2 \\
\hline
\end{tabular}

(a)

\begin{tabular}{|c|c|c|}
\hline 2 & 5 & -2 \\
\hline-8 & 6 & -4 \\
\hline 3 & -2 & 6 \\
\hline
\end{tabular}

(b)

\begin{tabular}{|c|c|c|}
\hline-3 & 6 & -2 \\
\hline-7 & 6 & -4 \\
\hline 6 & 5 & -1 \\
\hline
\end{tabular}

(e)

\begin{tabular}{|c|c|c|}
\hline-6 & 7 & 3 \\
\hline-5 & 6 & -6 \\
\hline 1 & 4 & 2 \\
\hline
\end{tabular}

(c)

\begin{tabular}{|l|l|l|}
\hline 0 & 1 & 2 \\
\hline 2 & 6 & 2 \\
\hline 1 & 2 & 1 \\
\hline
\end{tabular}

(f)

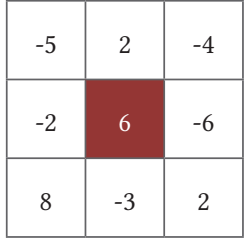

(d)

\begin{tabular}{|l|l|l|}
\hline 2 & 1 & 0 \\
\hline 2 & 6 & 2 \\
\hline 1 & 1 & 0 \\
\hline
\end{tabular}

(g)
Fig. 1. Calculation of LMePVEP patterns (a) Original 3x3 image block. (b) Forward first order derivative of the original image with $\mathrm{j}=1$ (c) Forward first order derivative of the original image with $\mathrm{j}=2$ (d) Backward first order derivative of the original image with $\mathrm{j}=1$ (e) Backward first order derivative of the original image with $\mathrm{j}=2$ (f) Combined LMePVEP values for $\mathrm{j}=1$ (g) Combined LMePVEP values for $\mathrm{j}=2$.

A $3^{*} 3$ image block is considered for the extraction of LMePVEP values as shown in Fig. 1(a). Forward first order derivative values 
for $j=1$ and $j=2$ are computed for the central pixel using eq. (1) and eq. (2) and these derivative values are shown in Fig. 1(b) and Fig. 1(c) respectively. Similarly, the backward first order derivative using for $j=1$ and $j=2$ are computed using eq. (3) as shown in Fig. 1(d) andFig. 1 (e) respectively. Then Combined LMePVEP coefficients are computed using eq. (5) which are shown in Fig. 1(f) and Fig. 1(g) for $j=1$ and $j=2$ respectively. These Combined LMePVEP values are then separated to obtain peak and valley patterns. Here pixels that contain value 2 represent peak and the pixels having values as 1 represent valley. Fig. 2(a) represents a matrix having valley information from Fig. 1(f). Such pixels having valley information (i.e. value 1 ) are assigned a value 1 and rest all are made zero. Similarly,Fig. 2(c) represents a matrix having peak information only. Matrix in Fig. 2(c) is obtained by considering pixels having value 2 in Fig. 1(f) and making the intensity of the rest of pixelsequal to 0 . In a similar manner we obtain the coded information of valley and peak in matrices of Fig. 2(b) and Fig. 2(d) from Fig. 1(g) for $\mathrm{j}=1$ and $\mathrm{j}=2$. A weight matrix $W$ consisting of $P$ values (in this case 8 ) is generated as shown in Fig. 2(e) using eq. (6) and an inner dot product of weight matrix and peak and valley values obtained in Fig. 2(a) to Fig. 2(d) is calculated. The sum of these individual results is used to replace the central shaded pixel. These values are final weighted LMePVEP values that replace the central pixel to obtain the final LMePVEP texture. This process is repeated for the entire image and all the pixels and finally four images based on weighted LMePVEP values are obtained.

$$
W(i)=1.2^{i-1} \quad \text { where } \mathrm{i}=1 \ldots \mathrm{P}
$$

Here for each image, we kept the value of $\mathrm{j}$ as 1 and 2 to obtain four corresponding images having LMePVEP texture information.

\begin{tabular}{|l|l|l|}
\hline 0 & 1 & 0 \\
\hline 0 & 6 & 0 \\
\hline 1 & 0 & 1 \\
\hline
\end{tabular}

(a)

\begin{tabular}{|l|l|l|}
\hline 1 & 0 & 0 \\
\hline 1 & 6 & 1 \\
\hline 0 & 0 & 0 \\
\hline
\end{tabular}

(d)

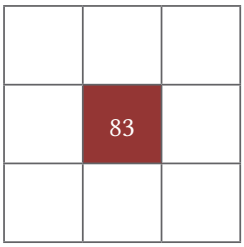

(g)

\begin{tabular}{|c|c|c|}
\hline 0 & 1 & 0 \\
\hline 0 & 6 & 0 \\
\hline 1 & 1 & 0 \\
\hline
\end{tabular}

(b)

\begin{tabular}{|c|c|c|}
\hline 8 & 4 & 2 \\
\hline 16 & & 1 \\
\hline 32 & 64 & 128 \\
\hline
\end{tabular}

(e)

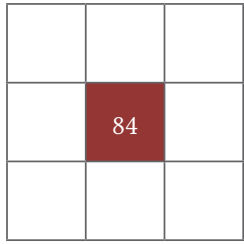

(h)

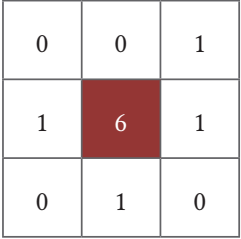

(c)

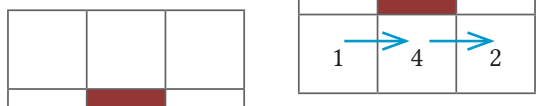

(b)

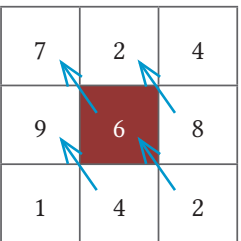

(e)

\begin{tabular}{|c|c|c|}
\hline 7 & 2 & 4 \\
\hline 7 & 2 & 8 \\
\hline-5 & -4 & 2 \\
\hline
\end{tabular}

(h)

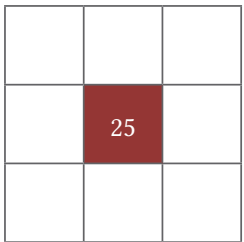

(i)
Fig. 2. (a) Local mesh valley pattern extracted from original image for $j=1$ (b) Local mesh valley pattern for $\mathrm{j}=2$ (c) Local mesh peak pattern for $\mathrm{j}=1$ (d) Local mesh peak pattern for $\mathrm{j}=2$ (e) Weight Matrix (f) Final Weighted LMePVEP value of central pixel for $j=1$ in forward direction (g)Weighted LMePVEP value of central pixel for $\mathrm{j}=1$ in backward direction (h)Weighted LMePVEP value of central pixel for $\mathrm{j}=2$ in forward direction (i)Weighted LMePVEP value of central pixel for $\mathrm{j}=2$ in backward direction.

\section{B. Texture in Various Directions}

Now we compute five different images based on the texture in five different directions for an image block given in Fig. 3.3(a). In [40], the texture in different directions is computed in the images obtained in section II. However, in this paper, we compute texture in original grayscale image. In the resultant images, one image will texture in one direction. To achieve that, for each case, the the difference of pixel intensity lying on the tail and on the head of the directional arrow, and these difference blocks are shown in Fig. 3(g) e values in each matrix of Fig. $3(\mathrm{~g})$ to Fig. $3(\mathrm{k})$ to obtain five (rices as shown in Fig. 3(1). These five central pixel values represent value of values present in matrix of Fig. 3(a). For the matrix in Fig. (a) the median value is 4 as shown in Fig. 4 . This median is taken to value as 1 and otherwise it will have a value 0 . Thus, the binarized abtional coefficients are obtained as shown in Fig. 3(m).

This process is continued for all overlapping $3 \times 3$ image blocks which are one pixel distant from each other in horizontal and vertical directions. Ultimately five binary images are obtained.

\begin{tabular}{|l|l|l|}
\hline 7 & 2 & 4 \\
\hline 9 & 6 & 8 \\
\hline 1 & 4 & 2 \\
\hline
\end{tabular}

(a)

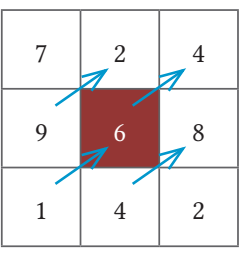

(c)

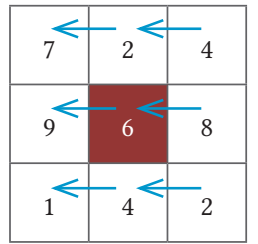

(f)

\begin{tabular}{|c|c|c|}
\hline 7 & 2 & 4 \\
\hline 2 & 4 & 4 \\
\hline-8 & -2 & -6 \\
\hline
\end{tabular}

(i)

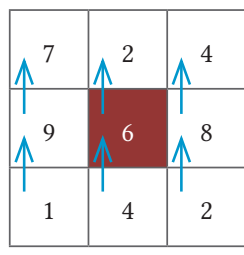

(d)

\begin{tabular}{|c|c|c|}
\hline 5 & -2 & 4 \\
\hline 3 & -2 & 8 \\
\hline-3 & 2 & 2 \\
\hline
\end{tabular}

(g)

\begin{tabular}{|c|c|c|}
\hline 7 & 2 & 4 \\
\hline 9 & -1 & 6 \\
\hline 1 & -5 & -4 \\
\hline
\end{tabular}

(j) 


\begin{tabular}{|c|c|c|}
\hline 7 & -5 & 2 \\
\hline 9 & -3 & 2 \\
\hline 1 & 3 & -2 \\
\hline
\end{tabular}

(k)
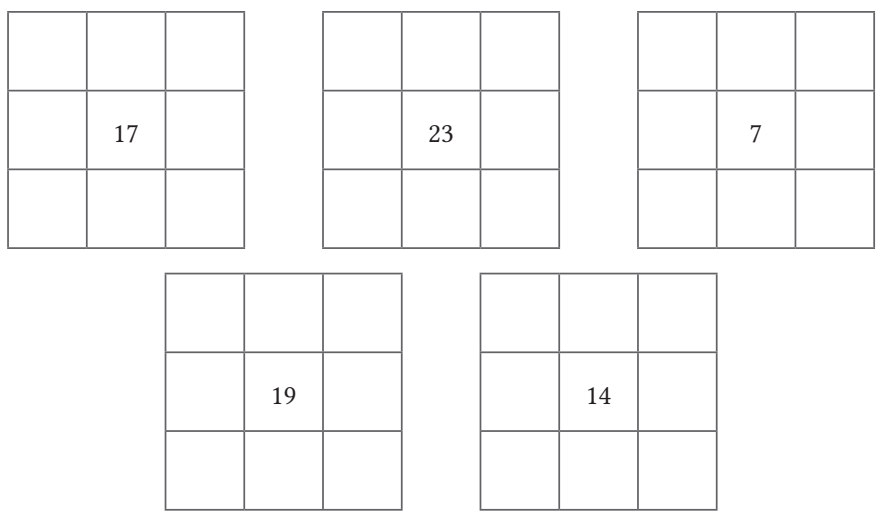

(l)
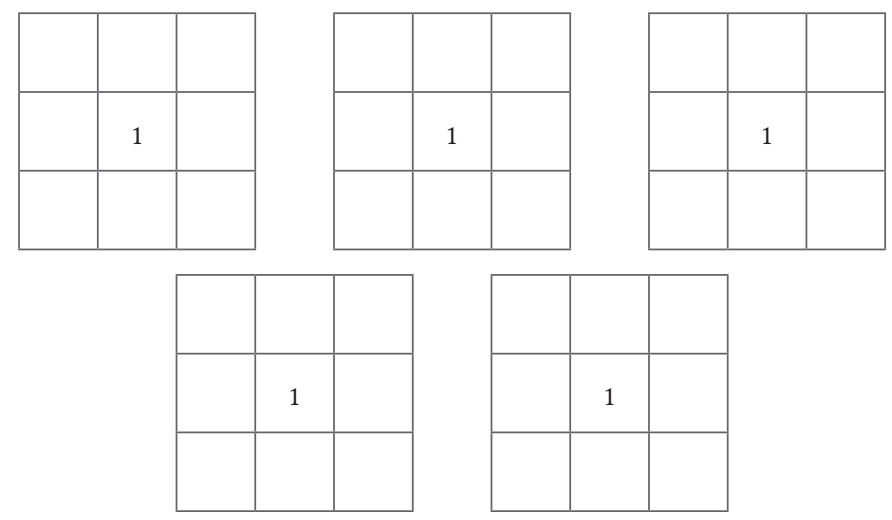

(m)

Fig. 3. (a) 3x3 input image block (b) pixel locations used to compute texture gradient in 0 degree (c) pixel locations used to compute texture gradient in 45 degree (d) pixel locations used to compute texture gradient in 90 degree (e) pixel locations used to compute texture gradient in 135 degree (f) pixel locations used to compute texture gradient in 180 degree (g) difference block for 0 degree (h) difference block for 45 degree (i) difference block for 90 degree $(\mathrm{j})$ difference block for 135 degree $(\mathrm{k})$ difference block for 180 degree (l) Summed up values for each matrix of Fig. 3(g) to Fig. 3(k) (m) Central pixel intensities for each direction obtained after comparing with a threshold value of 4 (median) to replace the central pixel intensity of 3x3 matrix in Fig. 3(a) and accordingly obtain five images.

\section{Dynamic Threshold Based Local Mesh Ternary Pattern}

This technique is derived from [39]. Here we compute the median of nine values in the $3 \times 3$ matrix extracted from an image. The median is taken as the threshold value for further computation.

For the computation of this feature vector, firstly Local Mesh Patterns (LMePs) are computed using eq. (7). This calculation is done up to $\mathrm{P} / 2$ values of $\mathrm{j}$.

$$
\operatorname{LMeP}_{(\mathrm{P}, \mathrm{R})}=\sum_{i=1}^{P} f_{l}\left(\left.g_{\alpha}\right|_{R}-\left.g_{i}\right|_{R}\right)
$$

Here $\alpha=1+\bmod ((i+P+j-1), P)$ for $j=1,2, \ldots$ up to $P / 2$.

Also $g$ represents the intensity value of a pixel, thus $\left.g_{\alpha}\right|_{R}=$ intensity value of $\alpha^{\text {th }}$ neighbor pixel (out of $P$ neighbors) at radius $R$ and $\left.g_{i}\right|_{R}=$ intensity value of the central pixel. Further function $f_{1}$ computes the difference between intensity value of $\left.g_{\alpha}\right|_{R}$ and $\left.g_{i}\right|_{R}$ pixel.

\begin{tabular}{|l|l|l|}
\hline 7 & 4 & 4 \\
\hline 9 & 6 & 8 \\
\hline 1 & 4 & 2 \\
\hline
\end{tabular}

(a)

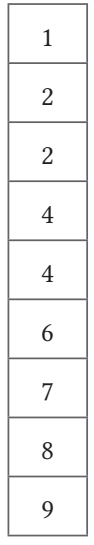

(b)

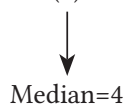

Fig. 4. Threshold computation as per [39] for a $3^{*} 3$ matrix from an image.

Then, if a matrix sub-component as shown in Fig. 5(a) is considered from an image, then after applying eq. (7), matrix components as given in Fig. 5(b) to Fig. 5(d) are obtained. Further, to compute the upper and lower ternary values, two thresholds are used. In this case, since the median value is 4, the two thresholds as given in [39] are two units greater and lesser than 4 . Thus, the two thresholds are taken as 6 and 2.

The values obtained in Fig. 5(b), Fig. 5(c) and Fig. 5(d) are then contrasted with thresholds 6 and 2 using eq. (8). If the values in these matrices aregreater than 6 , then +1 is the output, if the valuesareless than 2 , then -1 is output, and otherwise 0 is the output as given in matrices of Fig. 5(e), Fig. 5(f) and Fig. 5(g).

$$
\mathrm{f} 2(\mathrm{x}, \text { med., } \mathrm{t})=\left\{\begin{array}{l}
+1, \text { for } \mathrm{x} \geq \text { med. } \\
0, \text { for } \mid \mathrm{x} \text {-med. } \mid<\mathrm{t} \\
-1, \text { for } \mathrm{x} \leq \text { med. }-\mathrm{t}
\end{array}\right.
$$

Now the upper and lower LTPs are separated by separating the positive and negative values in matrices obtained in Fig. 5(e), Fig. 5(f) and Fig. 5(g) to obtain the six matrices of Fig. 5(h). For e.g. the matrix in fig. 5(e) is used to constitute two matrices, one matrix in which only positive values are considered and rest all are kept 0 , whereas the other matrix is constituted by keeping the negative values and rest all are made 0 . In this way, we obtain the six matrices of Fig. 5(h) from the three matrices of Fig. 5(e), Fig. 5(f) and Fig. 5(g).

Here $t=2$ and med. (median value) is 4 .

These are then multiplied with weight matrix in Fig. 5(i). The multiplied values are then summed up to replace the central pixel intensity of six matrices as shown in Fig. 5(j). This process is repeated for all pixels that are one pixel distant and accordingly we obtain six different images for a single image.

\begin{tabular}{|l|l|l|}
\hline 7 & 2 & 4 \\
\hline 9 & 6 & 8 \\
\hline 1 & 4 & 2 \\
\hline
\end{tabular}

(a) 


\begin{tabular}{|c|c|c|}
\hline 2 & 5 & -2 \\
\hline-8 & & -4 \\
\hline 3 & -2 & 6 \\
\hline
\end{tabular}

(b)

\begin{tabular}{|c|c|c|}
\hline-1 & 0 & -1 \\
\hline-1 & & -1 \\
\hline 0 & -1 & 1 \\
\hline
\end{tabular}

(e)

\begin{tabular}{|l|l|l|}
\hline 0 & 0 & 0 \\
\hline 0 & & 0 \\
\hline 0 & 0 & 1 \\
\hline
\end{tabular}

\begin{tabular}{|l|l|l|}
\hline 1 & 1 & 0 \\
\hline 1 & & 1 \\
\hline 1 & 0 & 1 \\
\hline
\end{tabular}

\begin{tabular}{|c|c|c|}
\hline 0 & 0 & 0 \\
\hline 0 & & 0 \\
\hline 1 & 0 & 0 \\
\hline
\end{tabular}

(h)

\begin{tabular}{|c|c|c|}
\hline 8 & 4 & 2 \\
\hline 16 & & 1 \\
\hline 32 & 64 & 128 \\
\hline
\end{tabular}

(i)
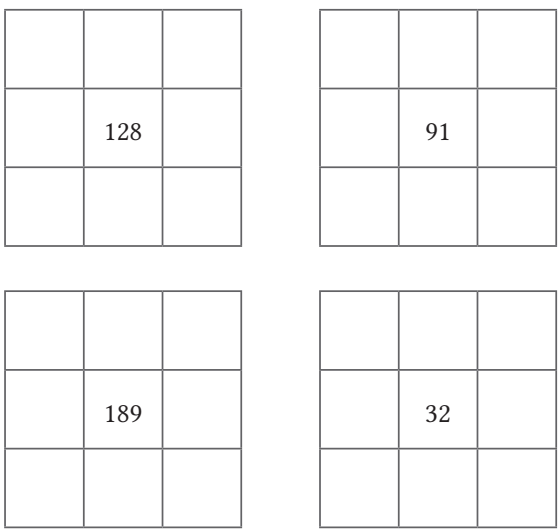

\begin{tabular}{|c|c|c|}
\hline-3 & -1 & 5 \\
\hline-7 & & -1 \\
\hline 7 & 0 & 0 \\
\hline
\end{tabular}

(d)

\begin{tabular}{|c|c|c|}
\hline-1 & -1 & 0 \\
\hline-1 & & -1 \\
\hline 1 & -1 & -1 \\
\hline
\end{tabular}

(g)

\begin{tabular}{|l|l|l|}
\hline 0 & 0 & 0 \\
\hline 0 & & 0 \\
\hline 0 & 1 & 0 \\
\hline
\end{tabular}

\begin{tabular}{|l|l|l|}
\hline 1 & 1 & 0 \\
\hline 1 & & 1 \\
\hline 0 & 1 & 1 \\
\hline
\end{tabular}

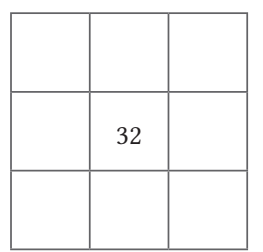

(j)
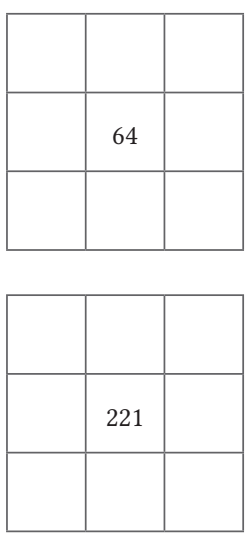

Fig. 5: Calculation of dynamic LMeTerP. (a) Intensity values in a $3 * 3$ sub-part of an image. LMePs for (b) $j=1$ (c) $j=2$ (d) $j=3$. LMeTerP values using function $f_{2}$ of eq.(8) for (e) $j=1$ (f) $j=2$ (g) $j=3$ (h) Extraction of Upper and Lower ternary values for Fig. 5(d-f) (i) A standard weight matrix (j) Final values of dynamic LMeTerP after dot product multiplication of values in Fig. 5(h) with values in Fig. 5(i) and then summation.

\section{Computation of Final Feature Vector}

In subsection $\mathrm{A}, \mathrm{B}$ and $\mathrm{C}$ of section II we obtain four, five and six images respectively for a single image. Thus, a total of fifteen images with texture details are obtained for a single image as given in Fig. 9. Histograms of these fifteen images are thereby computed and combined to form the feature vector.

The proposed feature extraction approach is unveiled in Fig. 6. The feature vector length comparison for different state of the art texturebased algorithms is summarized in Table I.

TABLE I. Comparison of Feature Vector Lengths of Various TextureBASEDCBIR MECHANISMS

\begin{tabular}{|c|c|}
\hline Method & Feature vector length \\
\hline LBP & 256 \\
\hline LTP & $2 \times 256$ \\
\hline LMePVEP & $4 \times 256$ \\
\hline LMeTerP & $3 \times 2 \times 256$ \\
\hline Proposed algorithm & $15 \times 256$ \\
\hline
\end{tabular}

The extracted set of features is then fed into a classifier to classify between various classes or severity levels of a disease. Two classifiers are used in the proposed methodology viz. back propagation neural networks $(\mathrm{BNN})$ and $\mathrm{k}$ nearest neighbors $(\mathrm{KNN})$. In back propagation neural network, the number of neurons in hidden layer has been kept to 10 .

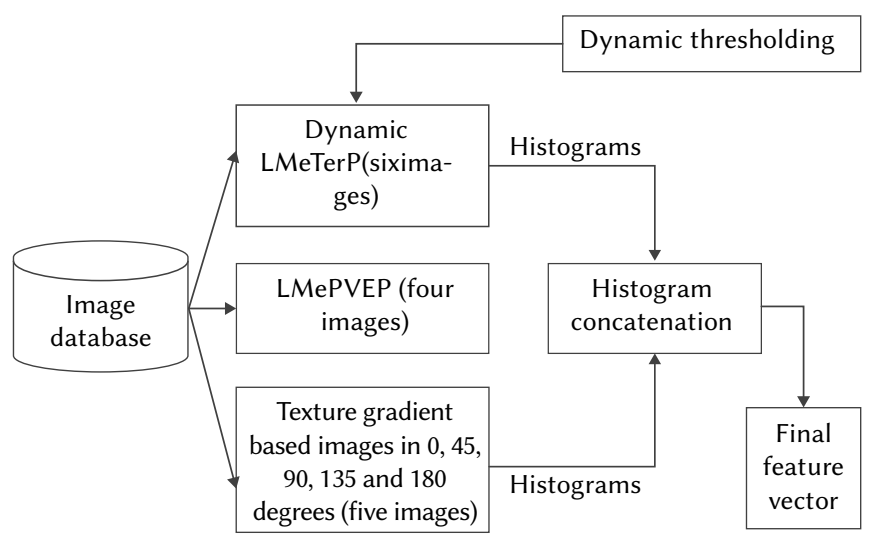

Fig. 6. Schematic representation of feature extraction.

\section{Results AND Discussion}

The proposed framework has been implemented using MATLAB version 2018a platform on a computational device with Windows environment. The computational device has a RAM of 8 GB and 2.0 $\mathrm{GHz}$ octa-core processor.

The results are evaluated using two databases. The first database is of Lung CT images for 39 subjects divided into two groups. One group is of no or minimal emphysema and another of mild, average, and high emphysema [36]. A total of 124 images were there in .tiff extension. Fig. 7 shows two CT-Scan images, one from each group.

The second dataset is also of CT images of lungs obtained from ELCAP repository [37]. The dataset had images with nodules present in different location of lungs. A total of 10 different groups are there as per different location of nodules. Fig. 8 shows one image from each group of ELCAP database. 


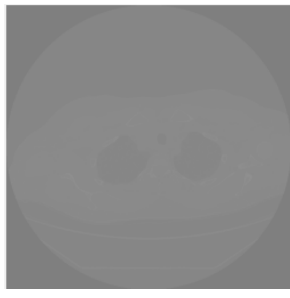

(a)

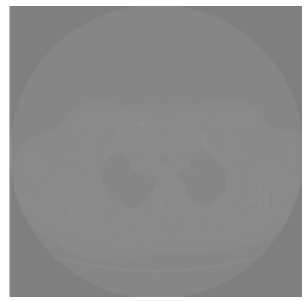

(b)

Fig. 7. CT images of patients with emphysema condition as - (a) minimal or no emphysema (b) High emphysema level.

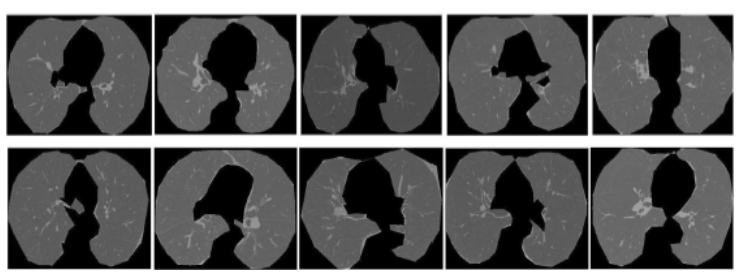

Fig. 8. Images obtained from ELCAP lung database.

Fifteen images are obtained when we apply the given techniques on a single image. We then generate the histograms of these images and concatenate them to form the final feature vector. All these fifteen images are given in Fig. 9 for a given original image from dataset 1.
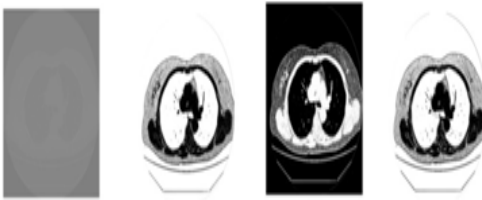

(a)
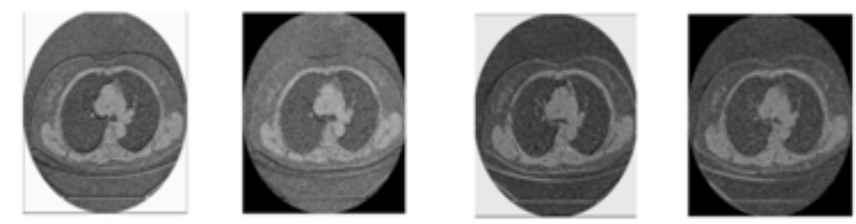

(b)
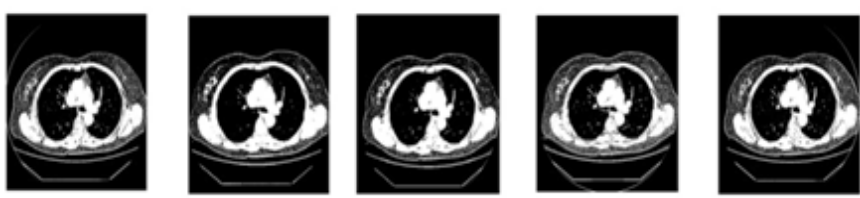

(c)

Fig. 9. (a) Image taken from emphysema database followed by six images obtained from dynamic threshold based LMeTerP. These six images are for $j=1$ for upper LTP, $j=1$ for lower LTP, $j=2$ for upper LTP, $j=2$ for lower LTP, $j=3$ for upper LTP and $j=3$ by for lower LTP respectively. (b) Four LMePVEP images for $j=1$ in forward direction, $j$ in backward direction, $j=2$ in forward direction and $\mathrm{j}=2$ in backward direction, respectively. (c) Five images obtained in $0,45,90,135$ and 180 degree direction respectively.

The feature vector is then fed into $\mathrm{KNN}$ or $\mathrm{BNN}$ for further classification as either an image for diseased individual or as an image for healthy individual. The feature vector of the proposed algorithm has been compared with feature vectors of Hybrid Intelligent Technique (HIT) technique for brain MR image classification [32], LMePVEP technique in [33], Dynamic LMeTerP technique of [39] and Shearlet Based Texture and Radial Basis Transform (SBT-RBF) [42]. The performance is analyzed using Accuracy metric as given in eq. (9) [32].

$$
\text { Accuracy }=\frac{\text { No.of } \text { True Positive samples }}{\text { Total number of samples }}
$$

Here the true positive samples are the ones that are correctly classified into their respective class. The feature vectors of four algorithms are analyzed on two classifiers viz. K-Nearest Neighbor $(\mathrm{KNN})$ and Back-propagation Neural Network (BNN). The comparison is done by varying percentage of images in testing and training dataset as given in Table II, Table III and table IV respectively.

TABLE II. Accuracy (IN \%) When the Dataset Is Split for 75 ANd 25 Percent Training and Testing Data

\begin{tabular}{|c|c|c|c|c|c|}
\hline Classifier & $\begin{array}{c}\text { HIT for } \\
\text { MR Image } \\
\text { classification }\end{array}$ & LMePVEP & $\begin{array}{l}\text { Dynamic } \\
\text { LMeTerP }\end{array}$ & $\begin{array}{l}\text { SBT- } \\
\text { RBF }\end{array}$ & $\begin{array}{l}\text { Prop. } \\
\text { Algo. }\end{array}$ \\
\hline \multicolumn{6}{|c|}{ Dataset 1} \\
\hline KNN & 75.1 & 81.2 & 85.2 & 91.3 & 92.5 \\
\hline BNN & 83.4 & 84.2 & 85.9 & 92.8 & 93.4 \\
\hline \multicolumn{6}{|c|}{ Dataset 2} \\
\hline KNN & 55.5 & 54.4 & 55.5 & 76.5 & 79.3 \\
\hline BNN & 65.6 & 67.3 & 67.8 & 70.4 & 74.2 \\
\hline
\end{tabular}

TABLE III. Accuracy (In \%) When the Dataset Is Split for 80 And 20 Percent Training and Testing Data

\begin{tabular}{|c|c|c|c|c|c|}
\hline Classifier & $\begin{array}{c}\text { HIT for } \\
\text { MR Image } \\
\text { classification }\end{array}$ & LMePVEP & $\begin{array}{l}\text { Dynamic } \\
\text { LMeTerP }\end{array}$ & $\begin{array}{l}\text { SBT- } \\
\text { RBF }\end{array}$ & $\begin{array}{l}\text { Prop. } \\
\text { Algo. }\end{array}$ \\
\hline \multicolumn{6}{|c|}{ Dataset 1} \\
\hline KNN & 81.1 & 87.2 & 91.3 & 93.4 & 95.3 \\
\hline BNN & 79.3 & 82.5 & 86.2 & 93.5 & 93.4 \\
\hline \multicolumn{6}{|c|}{ Dataset 2} \\
\hline KNN & 69.4 & 68.2 & 72.2 & 70.2 & 73.4 \\
\hline $\mathrm{BNN}$ & 64.4 & 66.9 & 68.3 & 71.4 & 74.2 \\
\hline
\end{tabular}

TABLE IV. Accuracy (In \%) When the Dataset Is Split for 85 AND 15 Percent Training and Testing Data

\begin{tabular}{|c|c|c|c|c|c|}
\hline Classifier & $\begin{array}{c}\text { HIT for } \\
\text { MR Image } \\
\text { classification }\end{array}$ & LMePVEP & $\begin{array}{l}\text { Dynamic } \\
\text { LMeTerP }\end{array}$ & $\begin{array}{l}\text { SBT- } \\
\text { RBF }\end{array}$ & $\begin{array}{l}\text { Prop. } \\
\text { Algo. }\end{array}$ \\
\hline \multicolumn{6}{|c|}{ Dataset 1} \\
\hline KNN & 84.5 & 83.1 & 84.3 & 90.5 & 91.2 \\
\hline BNN & 81.1 & 83.4 & 85.4 & 92.3 & 92.9 \\
\hline \multicolumn{6}{|c|}{ Dataset 2} \\
\hline KNN & 74.07 & 73.1 & 74.07 & 76.2 & 77.2 \\
\hline BNN & 65.0 & 66.3 & 66.7 & 72.8 & 73.5 \\
\hline
\end{tabular}

As we can see from Table II, table III and table IV, the feature vector of the proposed algorithm outperforms all the other algorithms which are used in the formation of its feature vector for all the cases. In all ratios of testing and training dataset and for both the classifiers the proposed feature vector is better in terms of accuracy.

\section{CONCLUSION}

The paper combines three different approaches of bio-medical image retrieval to propose a feature vector which is robust and enhances the accuracy of image retrieval. From section III, we can calculate the average accuracy for the proposed algorithm in the case of dataset 1 as $93.31 \%$ and that of algorithms HIT, LMePVEP, Dynamic LMeTerP 
and SBT-RBF to be $80.75 \%, 83.6 \%, 86.3 \%$ and $92.3 \%$ respectively. For dataset 2 these values are $65.66 \%, 66.03 \%, 67.4 \%, 72.91$, and $75.03 \%$ for algorithmsHIT, LMePVEP, Dynamic LMeTerP, SBT-RBF and the proposed algorithm, respectively.

Thereby the proposed framework has improved the accuracy by $12.56 \%, 9.71 \%, 7.01 \%$ and $1.01 \%$ in average for data set 1 and $9.37 \%$, $8.99 \%, 7.63 \%$ and $2.11 \%$ in average for dataset 2 over HIT, LMePVEP, Dynamic LMeTerP and SBT-RBFalgorithms respectively.

\section{REFERENCES}

[1] Eakins, John, and Margaret Graham. "Content-based image retrieval.", JISC technology education programme,1999.

[2] Deep, G., L. Kaur, and S. Gupta. "Local mesh ternary patterns: a new descriptor for MRI and CT biomedical image indexing and retrieval." Computer Methods in Biomechanics and Biomedical Engineering: Imaging \& Visualization, Vol. 6, no. 2, 2018, pp. 155-169.

[3] Haralick, Robert M., Karthikeyan Shanmugam, and Its' HakDinstein. "Textural features for image classification." IEEE Transactions on systems, man, and cybernetics, Vol. 6, 1973, pp. 610-621.

[4] Choi, Hyeokho, and Richard G. Baraniuk. "Multiscale image segmentation using wavelet-domain hidden Markov models.” IEEE Transactions on Image Processing, Vol. 10, no. 9, 2001, pp. 1309-1321.

[5] Ghosh, Payel, Sameer Antani, L. Rodney Long, and George R. Thoma "Review of medical image retrieval systems and future directions." In 24th International Symposium on Computer-Based Medical Systems (CBMS), pp. 1-6, 2011, IEEE.

[6] Zhang, L., Zhou, Z., \& Li, H., "Binary Gabor pattern: An efficient and robust descriptor for texture classification”. 19th IEEE International Conference on Image Processing (ICIP),2012,pp. 81-84.

[7] Nanni, L., Lumini, A., \&Brahnam, S., "Local binary patterns variants as texture descriptors for medical image analysis”. Artificial Intelligence in Medicine,Vol. 49(2), 2010, pp.117-125.

[8] Do, M., N., Vetterli, M., "Wavelet-based texture retrieval using generalized Gaussian density and Kullback-leibler distance". IEEE Trans. on Image Processing, Vol.11(2), 2002, pp. 146-158.

[9] Murala, Subrahmanyam, R. P. Maheshwari, and R. Balasubramanian. "Directional binary wavelet patterns for biomedical image indexing and retrieval.” Journal of Medical Systems, Vol. 36, no. 5,2012, pp. 2865-2879.

[10] Murala, Subrahmanyam, R. P. Maheshwari, and R. Balasubramanian. "Directional local extrema patterns: a new descriptor for content based image retrieval." International journal of multimedia information retrieval, Vol. 1, no. 3, 2012, pp. 191-203.

[11] Murala, S., Maheshwari, R., P., and Balasubramanian, R., "Local maximum edge binary patterns: a new descriptor for image retrieval and object tracking". Signal Processing, Vol. 92(6), 2012, pp.1467-1479.

[12] Ojala, Timo, Matti Pietikainen, and Topi Maenpaa. "Multiresolution gray-scale and rotation invariant texture classification with local binary patterns."IEEE Transactions on pattern analysis and machine intelligence, Vol.24.7, 2002, pp. 971-987.

[13] Tan, Xiaoyang, and Bill Triggs. "Enhanced local texture feature sets for face recognition under difficult lighting conditions." IEEE transactions on image processing, Vol. 19.6,2010, pp. 1635-1650.

[14] Rahman, Md Mahmudur, Prabir Bhattacharya, and Bipin C. Desai. "A framework for medical image retrieval using machine learning and statistical similarity matching techniques with relevance feedback." IEEE transactions on Information Technology in Biomedicine, Vol. 11.1, 2007, pp. 58-69.

[15] Rajeswari, J., and M. Jagannath. "Advances in biomedical signal and image processing-A systematic review." Informatics in Medicine Unlocked, Vol. 8, 2017, pp. 13-19.

[16] Galshetwar, Gajanan M., et al. "Edgy salient local binary patterns in interplane relationship for image retrieval in Diabetic Retinopathy." Procedia Computer Science, Vol. 115, 2017, pp. 440-447.

[17] Jyothi, B., MadhaveeLatha, Y., Mohan, P. K., \& Reddy, V. S. K.,"Integrated multiple features for tumor image retrieval using classifier and feedback methods",Procedia Computer Science, Vol. 85, 2016, pp.141-148.

[18] Ma, Ling, et al. "A new method of content based medical image retrieval and its applications to CT imaging sign retrieval.” Journal of biomedical informatics, Vol. 66, 2017, pp. 148-158.

[19] Xu, Songhua, and Michael Krauthammer. "A new pivoting and iterative text detection algorithm for biomedical images.” Journal of biomedical informatics, Vol. 43(6),2010, pp. 924-931.

[20] Farruggia, Alfonso, Rosario Magro, and Salvatore Vitabile. "A text based indexing system for mammographic image retrieval and classification.” Future Generation Computer Systems, Vol. 37,2014, pp. 243-251.

[21] Simpson, Matthew S., Daekeun You, Md Mahmudur Rahman, ZhiyunXue, Dina Demner-Fushman, Sameer Antani, and George Thoma. "Literaturebased biomedical image classification and retrieval." Computerized Medical Imaging and Graphics, Vol. 39, 2015, pp. 3-13.

[22] Charles, YesubaiRubavathi, and Ravi Ramraj. "A novel local mesh color texture pattern for image retrieval system.” AEU-International Journal of Electronics and Communications, Vol. 70(3),2016, pp. 225-233.

[23] Verma, Manisha, and Balasubramanian Raman. "Center symmetric local binary co-occurrence pattern for texture, face and bio-medical image retrieval." Journal of Visual Communication and Image Representation, Vol.32,2015, pp.224-236.

[24] Kumar, Yogesh, et al. "An efficient and robust approach for biomedical image retrieval using Zernike moments.” Biomedical Signal Processing and Control, Vol. 39, 2018, pp.459-473.

[25] Singh, Vibhav Prakash, and Rajeev Srivastava. "Automated and effective content-based mammogram retrieval using wavelet based CS-LBP feature and self-organizing map." Biocybernetics and Biomedical Engineering, Vol. 38(1),2018, pp.90-105.

[26] Deep, G., L. Kaur, and S. Gupta. "Directional local ternary quantized extrema pattern: A new descriptor for biomedical image indexing and retrieval." Engineering Science and Technology, an International Journal, Vol. 19(4), 2016, pp. 1895-1909.

[27] Tiwari, Ashwani Kumar, Vivek Kanhangad, and Ram Bilas Pachori. "Histogram refinement for texture descriptor based image retrieval.” Signal Processing: Image Communication, Vol 53,2017, pp. 7385.

[28] Li, Wenbo, Haiwei Pan, Pengyuan Li, XiaoqinXie, and Zhiqiang Zhang. "A medical image retrieval method based on texture block coding tree." Signal Processing: Image Communication, Vol. 59, 2017, pp. 131-139.

[29] Murala, Subrahmanyam, and QM Jonathan Wu. "Spherical symmetric 3D local ternary patterns for natural, texture and biomedical image indexing and retrieval.” Neurocomputing, Vol. 49 (1), 2015, pp. 1502-1514.

[30] Qayyum, Adnan, Syed Muhammad Anwar, Muhammad Awais, and Muhammad Majid. "Medical image retrieval using deep convolutional neural network.” Neurocomputing, Vol. 266, 2017, pp.8-20.

[31] Alzu'bi, Ahmad, Abbes Amira, and Naeem Ramzan. "Content-based image retrieval with compact deep convolutional features." Neurocomputing, Vol. 249, 2017, pp.95-105.

[32] El-Dahshan, El-Sayed Ahmed, Hosny, Tamer and Salem Abdel-Badeeh M, Hybrid intelligent techniques for MRI brain images classification, Digital Signal Processing, Vol. 20(2), 2010, pp.433-441, Elsevier.

[33] Murala, Subrahmanyam, and QM Jonathan Wu. "MRI and CT image indexing and retrieval using local mesh peak valley edge patterns." Signal Processing: Image Communication, Vol. 29(3), 2014, pp.400-409.

[34] S. Varun, and R. K.Purwar, "A Five-Level Wavelet Decomposition and Dimensional Reduction Approach for Feature Extraction and Classification of MR and CT Scan Images," Applied Computational Intelligence and Soft Computing, Vol. 2017, Article ID 9571262,2017,.doi: https://doi.org/10.1155/2017/9571262

[35] Purwar, Ravindra Kr, and Varun Srivastava. "Recent Advancements in Detection of Cancer Using Various Soft Computing Techniques for MR Images." Progress in Advanced Computing and Intelligent Engineering. Springer, Singapore,pp.99-108, 2018.

[36] Emphysema dataset, L. Sørensen, S. B. Shaker, and M. de Bruijne, "Quantitative Analysis of Pulmonary Emphysema using Local Binary Patterns”, IEEE Transactions on Medical Imaging, Vol. 29(2), 2010, pp. 559-569.

[37] ELCAP dataset, A. P. Reeves, A. M. Biancardi, D. Yankelevitz, S. Fotin, B. M. Keller, A. Jirapatnakul, J. Lee. "A Public Image Database to Support Research in Computer Aided Diagnosis,' In 31st Annual International Conference of the IEEE Engineering in Medicine and Biology Society, pp. 3715-3718, Sept. 2009. 
[38] Sverzellati,Nicola,DavidA.Lynch,MassimoPistolesi,Hans-UlrichKauczor, Phillippe A. Grenier, Carla Wilson, and James D. Crapo. "Physiologic and quantitativecomputedtomographydifferencesbetweencentrilobular and panlobularemphysemainCOPD."ChronicObstructivePulmonaryDiseases: Journal of the COPDFoundation, Vol. 1(1), 2014, p. 125.

[39] Srivastava Varun, Ravindra K. Purwar, and Anchal Jain. "A dynamic threshold-based local mesh ternary pattern technique for biomedical image retrieval." International Journal of Imaging Systems and Technology, Vol. 29(2),2019, pp. 168-179.

[40] Srivastava Varun and Ravindra Purwar. "An extension of local mesh peak valley edge based feature descriptor for image retrieval in bio-medical images." ADCAIJ: Advances in Distributed Computing and Artificial Intelligence Journal, Vol. 7(1),2018, pp.77-89.

[41] Srivastava, Varun, and Ravindra Kr Purwar. "Classification of CT scan images of lungs using deep convolutional neural network with external shape-based features." Journal of digital imaging, Vol. 33(1), 2020, pp.252261.

[42] Semunigus, Wogderes. "Pulmonary Emphysema Analysis using Shearlet based textures and radial basis function network" International Journal of Advances in Signal and Image Sciences, Vol. 6(1), 2020, pp: 1-11.

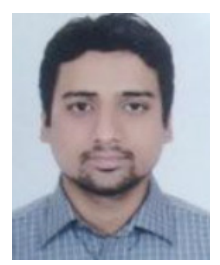

\section{Varun Srivastava}

Mr. Varun Srivastava is currently associated as an Assistant Professor with Computer Science Department of Bharati Vidyapeeth's College of Engineering. His main research areas are Biomedical Image Processing and Pattern Recognition. He has many research articles to his credit in these domains.

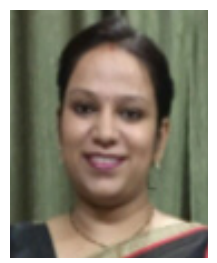

\section{Shilpa Gupta}

Ms. Shilpa Gupta is currently associated with Computer Science Department of Bharati Vidyapeeth'College of Engineering as an Assistant Professor. Her main areas of research are Machine learning and Deep networks. She has contributed many renowned publications in these domains.

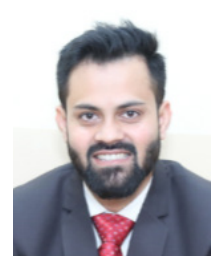

\section{Gopal Chaudhary}

Dr. Gopal Chaudhary is currently working as an assistant professor in Bharati Vidyapeeth's College of Engineering, Guru Gobind Singh Indraprastha University, Delhi, India. He holds a Ph.D. in Biometrics at the division of Instrumentation and Control engineering, Netaji Subhas Institute of Technology, University of Delhi, India. He received the B.E. degree in electronics and communication engineering in 2009 and the M.Tech. degree in Microwave and optical communication from Delhi Technological University (formerly known as Delhi College of Engineering), New Delhi, India, in 2012. He has 30 publications in refereed National/International Journals \& Conferences (Elsevier, Springer, Inderscience) in the area of Biometrics and its applications. His current research interests include soft computing, intelligent systems, information fusion and pattern recognition. He has organized many conferences and special issues.

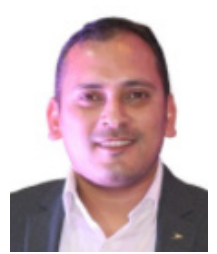

\section{Arun Balodi}

Dr. Arun Balodi has done his Ph. D. from Indian Institute of Technology Roorkee and is currently heading the Electronics and Communication Engineering Department of Atria Institute of Technology, Bengaluru. His main areas of research are Image processing and Machine learning.

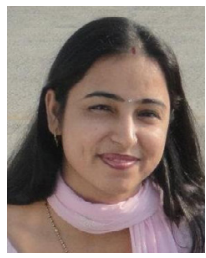

\section{Manju Khari}

Dr. Manju Khari is an Assistant Professor in Netaji Subhas University of Technology, East Campus, Delhi, India. She is also the Professor- In-charge of the IT Services of the Institute and has experience of more than twelve years in Network Planning \& Management. She holds a Ph.D. in Computer Science \& Engineering from National Institute Of Technology Patna and She received her master's degree in Information Security from Ambedkar Institute of Advanced Communication Technology and Research, formally this institute is known as Ambedkar Institute Of Technology affiliated with Guru Gobind Singh Indraprastha University, Delhi, India. Her research interests are software testing, information security, optimization, Image processing and machine learning. She has 70 published papers in refereed National/International Journals \& Conferences (viz. IEEE, ACM, Springer, Inderscience, and Elsevier) and 10+ edited books from reputed publishers. She is also co-author of two books published by NCERT of Secondary and senior Secondary School.

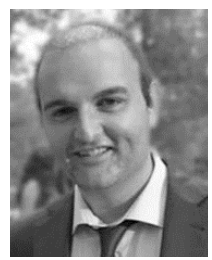

Vicente García-Díaz

Dr. Vicente García-Díaz is a Software Engineer, PhD in Computer Science. He has a Master in Occupational Risk Prevention. He has the qualification of University Expert in Blockchain Application Development. He is an Associate Professor in the Department of Computer Science at the University of Oviedo. He is also part of the editorial and advisory board of several journals and has been editor of several special issues in books and journals. He has supervised 100+ academic projects and published $100+$ research papers in journals, conferences, and books. His teaching interests are primarily in the design and analysis of algorithms and the design of domain-specific languages. His current research interests include machine learning, decision support systems, eHealth and eLearning. 